\title{
New Neighborhood based Rough Sets
}

\author{
Lynn D'eer ${ }^{1}$ and Chris Cornelis ${ }^{1,2}$ \\ 1 Department of Applied Mathematics, Computer Science and Statistics, \\ Ghent University, Belgium \\ lynn.deer@ugent.be \\ 2 Department of Computer Science and Artificial Intelligence, \\ University of Granada, Spain \\ chriscornelis@ugr.es
}

\begin{abstract}
Neighborhood based rough sets are important generalizations of the classical rough sets of Pawlak, as neighborhood operators generalize equivalence classes. In this article, we introduce nine neighborhood based operators and we study the partial order relations between twenty-two different neighborhood operators obtained from one covering. Seven neighborhood operators result in new rough set approximation operators. We study how these operators are related to the other fifteen neighborhood based approximation operators in terms of partial order relations, as well as to seven non-neighborhood-based rough set approximation operators.
\end{abstract}

Keywords: Neighborhood Operator, Rough Sets, Approximation Operator, Covering

\section{Introduction}

Pawlak [4] introduced rough set theory in order to deal with uncertainty in data systems due to incompleteness. Originally, an equivalence relation on the universe of discourse was used to describe the indiscernibility between elements. A generalization of rough sets is obtained by replacing the equivalence relation by a neighborhood operator (e.g. [11]). Another generalization results from considering a covering instead of the partition of equivalence classes ([12]).

In [3], twenty-four neighborhood operators obtained from one covering were studied. It was shown that the collection reduces to thirteen different operators. Moreover, it was discussed that two rough set approximation operators which were originally not introduced regarding a neighborhood operator are actually neighborhood based. In particular, they are related to the inverse operators of two of the thirteen neighborhood operators. In this article, we continue the study on inverse neighborhood operators in order to obtain new rough set approximation operators. We discuss nine inverse neighborhood operators, of which seven result in new rough set approximation operators. Furthermore, we study partial order relations between twenty-two neighborhood operators on the one hand, on the other hand partial order relations between twenty-nine approximation operators. Partial order relations between different approximation operators yield 
information on the accuracy of the approximation operators, i.e., upper approximation operators which yield smaller values will achieve higher accuracy, and therefore, they induce more accurate approximations of a concept.

The outline of this article is as follows. In Section 2, we present preliminary concepts. In Section 3, we introduce nine neighborhood operators and discuss the partial order relations of twenty-two neighborhood operators. In Section 4, the partial order relations of seven new approximation operators with existing rough set approximation operators are discussed. Finally, conclusions and future work are outlined in Section 5.

\section{Preliminaries}

Throughout this paper we assume that the universe $U$ is a non-empty set. Originally, Pawlak [4] used an equivalence relation $E$ to discern elements of the universe of discourse. Two fundamental concepts in rough set theory are the lower and upper approximation of a set $A \subseteq U$. These concepts describe the elements certainly and possibly belonging to $A$ :

$$
\begin{aligned}
& \underline{\operatorname{apr}}_{E}(A)=\left\{x \in U \mid[x]_{E} \subseteq A\right\}=\bigcup\left\{[x]_{E} \in U / E \mid[x]_{E} \subseteq A\right\}, \\
& \overline{\operatorname{apr}}_{E}(A)=\left\{x \in U \mid[x]_{E} \cap A \neq \emptyset\right\}=\bigcup\left\{[x]_{E} \in U / E \mid[x]_{E} \cap A \neq \emptyset\right\},
\end{aligned}
$$

where $U / E$ represents the set of equivalence classes defined from $E$. The first equality in Equations (1) and (2) is sometimes called the element based definition, while the second one is called the granule based definition [10].

Many generalizations of Pawlak's rough set model can be found in literature. In [10], a survey on dual generalizations is presented. A pair of approximation operators is called dual, if for all $A \subseteq U, \overline{\operatorname{apr}}(\operatorname{co}(A))=\operatorname{co}(\operatorname{apr}(A))$.

Equivalence classes can be generalized by neighborhood operators. A neighborhood operator $N$ is a mapping $N: U \rightarrow \mathcal{P}(U)$, where $\mathcal{P}(U)$ represents the collection of subsets of $U$. It is often assumed that the neighborhood operator is reflexive, i.e., $x \in N(x)$ for all $x \in U$. Given $N$, we define its inverse neighborhood operator $N^{-1}$ by $x \in N^{-1}(y) \Leftrightarrow y \in N(x)$ for $x, y \in U$.

Each neighborhood operator $N$ yields a dual pair of approximation operators $\left(\underline{\operatorname{apr}}_{N}, \overline{\operatorname{apr}}_{N}\right)$ defined by

$$
\begin{aligned}
& \underline{\operatorname{apr}}_{N}(A)=\{x \in U \mid N(x) \subseteq A\}, \\
& \overline{\operatorname{apr}}_{N}(A)=\{x \in U \mid N(x) \cap A \neq \emptyset\},
\end{aligned}
$$

for $A \subseteq U$ ([10]). The approximation operators generalize the element based definition stated in Equations (1) and (2). A particular neighborhood of $x$ is the afterset $R(x)$ of $x$ determined by a binary relation $R: R(x)=\{y \in U \mid x R y\}$ ([11]).

Generalization of the granule based approximation operators can be obtained by replacing the partition $U / E$ by a covering of $U$. Let $I$ be an index set, then 


\begin{tabular}{llll}
\hline Group & Operators & Group Operators \\
\hline$A$. & $N_{1}^{\mathbb{C}}, N_{1}^{\mathbb{C}_{1}}, N_{1}^{\mathbb{C}_{3}}, N_{2}^{\mathbb{C}_{3}}, N_{1}^{\mathbb{C}_{n}} \mid H$. & $N_{4}^{\mathbb{C}}, N_{2}^{\mathbb{C}_{2}}, N_{4}^{\mathbb{C}_{2}}, N_{4}^{\mathbb{C}_{n}}$ \\
\hline$B$. & $N_{3}^{\mathbb{C}_{1}}$ & $\mid I$. & $N_{2}^{\mathbb{C}_{4}}$ \\
\hline$C$. & $N_{3}^{\mathbb{C}_{3}}$ & $\mid J$. & $N_{3}^{\mathbb{C}_{4}}$ \\
\hline$D$. & $N_{4}^{\mathbb{C}_{3}}$ & $\mid K$. & $N_{4}^{\mathbb{C}_{4}}$ \\
\hline$E$. & $N_{2}^{\mathbb{C}}, N_{2}^{\mathbb{C}_{1}}$ & $N_{4}^{\mathbb{C}_{1}}$ \\
\hline$F$. & $N_{3}^{\mathbb{C}}, N_{1}^{\mathbb{C}_{2}}, N_{3}^{\mathbb{C}_{2}}, N_{3}^{\mathbb{C}_{n}}$ & $M$. & $N_{2}^{\mathbb{C}_{n}}$ \\
\hline$G$. & $N_{1}^{\mathbb{C}_{4}}$ & & \\
\hline
\end{tabular}

Table 1: Neighborhood operators based on coverings

a collection $\mathbb{C}=\left\{K_{i} \subseteq U \mid i \in I\right\}$ of non-empty subsets of $U$ is called a covering of $U$ if $\bigcup_{i \in I} K_{i}=U$.

Given a covering $\mathbb{C}$, we are interested in the sets $K \in \mathbb{C}$ such that $x \in K$. Let $\mathbb{C}$ be a covering of $U$ and $x \in U$, then the neighborhood system $\mathcal{C}(\mathbb{C}, x)$ of $x$ is defined by $\mathcal{C}(\mathbb{C}, x)=\{K \in \mathbb{C} \mid x \in K\}$ ([10]). Moreover, the sets

$$
\begin{aligned}
\operatorname{md}(\mathbb{C}, x) & =\{K \in \mathcal{C}(\mathbb{C}, x) \mid(\forall S \in \mathcal{C}(\mathbb{C}, x))(S \subseteq K \Rightarrow K=S)\}, \\
\operatorname{MD}(\mathbb{C}, x) & =\{K \in \mathcal{C}(\mathbb{C}, x) \mid(\forall S \in \mathcal{C}(\mathbb{C}, x))(S \supseteq K \Rightarrow K=S)\}
\end{aligned}
$$

are called the minimal [1] and maximal [14] description of $x$. They can be seen as the two extreme neighborhoods of $x$. If we apply the intersection and union to these neighborhood systems, we derive four neighborhood operators based on coverings [10]:

1. $N_{1}^{\mathbb{C}}(x)=\bigcap\{K \in \mathbb{C} \mid K \in \operatorname{md}(\mathbb{C}, x)\}=\bigcap \mathcal{C}(\mathbb{C}, x)$,

2. $N_{2}^{\mathbb{C}}(x)=\bigcup\{K \in \mathbb{C} \mid K \in \operatorname{md}(\mathbb{C}, x)\}$,

3. $N_{3}^{\mathbb{C}}(x)=\bigcap\{K \in \mathbb{C} \mid K \in \operatorname{MD}(\mathbb{C}, x)\}$,

4. $N_{4}^{\mathbb{C}}(x)=\bigcup\{K \in \mathbb{C} \mid K \in \operatorname{MD}(\mathbb{C}, x)\}=\bigcup \mathcal{C}(\mathbb{C}, x)$.

In addition, [10] considered five different coverings derived from a covering $\mathbb{C}$ :

1. $\mathbb{C}_{1}=\bigcup\{\operatorname{md}(\mathbb{C}, x) \mid x \in U\}$,

2. $\mathbb{C}_{2}=\bigcup\{\operatorname{MD}(\mathbb{C}, x) \mid x \in U\}$,

3. $\mathbb{C}_{3}=\{\bigcap \operatorname{md}(\mathbb{C}, x) \mid x \in U\}=\{\bigcap \mathcal{C}(\mathbb{C}, x) \mid x \in U\}$,

4. $\mathbb{C}_{4}=\{\bigcup \operatorname{MD}(\mathbb{C}, x) \mid x \in U\}=\{\bigcup \mathcal{C}(\mathbb{C}, x) \mid x \in U\}$,

5. $\mathbb{C}_{\cap}=\mathbb{C} \backslash\left\{K \in \mathbb{C} \mid\left(\exists \mathbb{C}^{\prime} \subseteq \mathbb{C} \backslash\{K\}\right)\left(K=\bigcap \mathbb{C}^{\prime}\right)\right\}$.

When combining the four neighborhood operators and the six coverings (one original and five derived ones), then we obtain twenty-four combinations $N_{i}^{\mathbb{C}_{j}}$ for $N_{i} \in\left\{N_{1}, N_{2}, N_{3}, N_{4}\right\}$ and $\mathbb{C}_{j} \in\left\{\mathbb{C}, \mathbb{C}_{1}, \mathbb{C}_{2}, \mathbb{C}_{3}, \mathbb{C}_{4}, \mathbb{C}_{\cap}\right\}$. However, in [3], it is stated that there are only thirteen different groups of neighborhood operators. These groups are shown in Table 1. Besides equalities between neighborhood operators, [3] studied partial order relations between the operators. Hence, the 


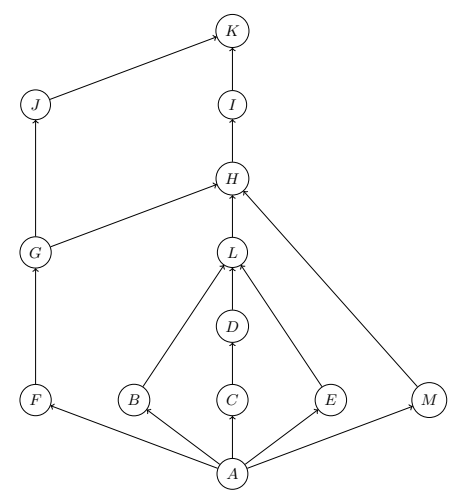

(a) Lattice of the neighborhood operators

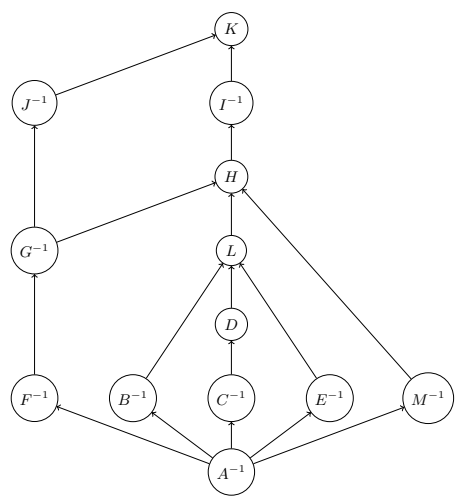

(b) Lattice of the inverse neighborhood operators

lattice given in Figure 1a with respect to the partial order relation $\leq$ was established: let $N$ and $N^{\prime}$ be two neighborhood operators, then $N \leq N^{\prime}$ if and only if $\forall x, y \in U: x \in N(y) \Rightarrow x \in N^{\prime}(y)$.

Moreover, it was stated that other covering based approximation operators which are not originally defined according to Equations (3) and (4), can be described with neighborhood operators. Let $\mathbb{C}$ be a covering, then the upper approximation operators $H_{3}^{\mathbb{C}}[8]$ and $H_{5}^{\mathbb{C}}$ [13] are defined as follows, let $A \subseteq U$, then

$$
\begin{aligned}
& H_{3}^{\mathbb{C}}(A)=\bigcup\left\{N_{2}^{\mathbb{C}}(x): x \in A\right\}, \\
& H_{5}^{\mathbb{C}}(A)=\bigcup\left\{N_{1}^{\mathbb{C}}(x): x \in A\right\} .
\end{aligned}
$$

We have the following proposition:

Proposition 1. [3] Let $\mathbb{C}$ be a covering and $A \subseteq U$, then

$$
\begin{aligned}
& H_{3}^{\mathbb{C}}(A)=\left\{x \in U \mid\left(N_{2}^{\mathbb{C}}\right)^{-1}(x) \cap A \neq \emptyset\right\}=\overline{\operatorname{apr}}_{\left(N_{2}^{\mathbb{C}}\right)^{-1}}(A), \\
& H_{5}^{\mathbb{C}}(A)=\left\{x \in U \mid\left(N_{1}^{\mathbb{C}}\right)^{-1}(x) \cap A \neq \emptyset\right\}=\overline{\operatorname{apr}}_{\left(N_{1}^{\mathbb{C}}\right)^{-1}}(A) .
\end{aligned}
$$

Therefore, $H_{3}^{\mathbb{C}}$ and $H_{5}^{\mathbb{C}}$ are element based approximation operators, based on the inverse neighborhood operators of $N_{2}^{\mathbb{C}}$ and $N_{1}^{\mathbb{C}}$ respectively.

\section{$3 \quad$ Neighborhood Operators}

Proposition 1 suggests that it is interesting to study the inverse neighborhood operators of the operators stated in Table 1. In this section, we discuss which inverse neighborhood operators are new. Moreover, we study the partial order relations and add them to Figure 1a. 


\begin{tabular}{|c|c|c|c|}
\hline \multicolumn{2}{|c|}{ Group Operators } & \multicolumn{2}{|c|}{ Group Operators } \\
\hline$A$. & $N_{1}^{\mathbb{C}}, N_{1}^{\mathbb{C}_{1}}, N_{1}^{\mathbb{C}_{3}}, N_{2}^{\mathbb{C}_{3}}, N_{1}^{\mathbb{C}_{n}}$ & $\mid A^{-1}$ & $\left(N_{1}^{\mathbb{C}}\right)^{-1},\left(N_{1}^{\mathbb{C}_{1}}\right)^{-1},\left(N_{1}^{\mathbb{C}_{3}}\right)^{-1},\left(N_{2}^{\mathbb{C}_{3}}\right)^{-1},\left(N_{1}^{\mathbb{C}_{n}}\right)^{-1}$ \\
\hline$B$. & $N_{3}^{\mathbb{C}_{1}}$ & $B^{-1}$ & $\left(N_{3}^{\mathbb{C}_{1}}\right)^{-1}$ \\
\hline$C$. & $N_{3}^{\mathbb{C}_{3}}$ & $\mid C^{-1}$. & $\left(N_{3}^{\mathbb{C}_{3}}\right)^{-1}$ \\
\hline$D$. & $N_{4}^{\mathbb{C}_{3}}$ & & \\
\hline$E$. & $N_{2}^{\mathbb{C}}, N_{2}^{\mathbb{C}_{1}}$ & $E^{-1}$ & $\left(N_{2}^{\mathbb{C}}\right)^{-1},\left(N_{2}^{\mathbb{C}_{1}}\right)^{-1}$ \\
\hline$F$. & $N_{3}^{\mathbb{C}}, N_{1}^{\mathbb{C}_{2}}, N_{3}^{\mathbb{C}_{2}}, N_{3}^{\mathbb{C}_{n}}$ & $F^{-1}$ & $\left(N_{3}^{\mathbb{C}}\right)^{-1},\left(N_{1}^{\mathbb{C}_{2}}\right)^{-1},\left(N_{3}^{\mathbb{C}_{2}}\right)^{-1},\left(N_{3}^{\mathbb{C}} \cap\right)^{-1}$ \\
\hline G. & $N_{1}^{\mathbb{C}_{4}}$ & $\mid G^{-1}$. & $\left(N_{1}^{\mathbb{C}_{4}}\right)^{-1}$ \\
\hline$H$. & $N_{4}^{\mathbb{C}}, N_{2}^{\mathbb{C}_{2}}, N_{4}^{\mathbb{C}_{2}}, N_{4}^{\mathbb{C}_{n}}$ & & \\
\hline$I$. & $N_{2}^{\mathbb{C}_{4}}$ & $I^{-1}$ & $\left(N_{2}^{\mathbb{C}_{4}}\right)^{-1}$ \\
\hline$J$. & $N_{3}^{\mathbb{C}_{4}}$ & $\mid J^{-1}$ & $\left(N_{3}^{\mathbb{C}_{4}}\right)^{-1}$ \\
\hline$K$. & $N_{4}^{\mathbb{C}_{4}}$ & | & \\
\hline$L$. & $N_{4}^{\mathbb{C}_{1}}$ & & \\
\hline$M$. & $N_{2}^{\mathbb{C}_{n}}$ & $M^{-1}$. & $\left(N_{2}^{\mathbb{C}_{n}}\right)^{-1}$ \\
\hline
\end{tabular}

Table 2: Neighborhood operators based on coverings and their inverses

\subsection{Inverse Neighborhood Operators}

To study the inverse operators of the thirteen neighborhood operators from Table 1 , we first observe that the operators $N_{4}^{\mathbb{C}_{j}}$ are symmetric, and therefore, they are equal to their inverses: e.g. let $x, y \in U$, then $x \in N_{4}^{\mathbb{C}_{j}}(y)$ if and only if there exists a $K \in \mathbb{C}_{j}$ such that $x, y \in K$. Hence, $y \in N_{4}^{\mathbb{C}_{j}}(x)$, or $x \in\left(N_{4}^{\mathbb{C}_{j}}\right)^{-1}(y)$.

Moreover, the inverses of two different operators are different as well.

Proposition 2. Let $N$ and $N^{\prime}$ be two different neighborhood operators, then $N^{-1} \neq N^{\prime-1}$.

Proof. If $N \neq N^{\prime}$, then there exist $x, y \in U$ such that $y \in N(x)$ and $y \notin N^{\prime}(x)$. Hence, $x \in N^{-1}(y)$ and $x \notin N^{\prime-1}(y)$. Thus, $N^{-1} \neq N^{\prime-1}$.

Combining the above two observations, there are nine possible new neighborhood operators. We need to check whether any of the inverse operators coincides with one of the thirteen known groups. However, this is not the case. Here we illustrate that the operator $A^{-1}$ is different from the operators $A-D$. Other counterexamples can be obtained similarly.

Example 1. Let $U=\{1,2,3\}$ and $\mathbb{C}=\{\{1,2\},\{1,2,3\}\}$. Then $\left(N_{1}^{\mathbb{C}}\right)^{-1}(3)=\{3\}$, but $N_{1}^{\mathbb{C}}(3)=N_{3}^{\mathbb{C}_{1}}(3)=N_{3}^{\mathbb{C}_{3}}(3)=N_{4}^{\mathbb{C}_{3}}(3)=\{1,2,3\}$. Therefore, the neighborhood operators of $A^{-1}$ are different than the ones of $A, B, C$ and $D$.

Hence, we obtain twenty-two neighborhood operators stated in Table 2. For each group $X$, we denote $X^{-1}$ for the group of inverse operators from $X$. 


\subsection{Partial Order Relation for Neighborhood Operators}

The objective of this section is to obtain a Hasse diagram containing the twentytwo neighborhood operators from Table 2 with respect to the partial order relation $\leq$. First, we present the following proposition:

Proposition 3. Let $N$ and $N^{\prime}$ be two neighborhood operators such that $N \leq N^{\prime}$, then $N^{-1} \leq N^{\prime-1}$.

Proof. Let $N \leq N^{\prime}$, then for all $x, y \in U$ it holds that $y \in N(x) \Rightarrow y \in N^{\prime}(x)$. Hence, $x \in N^{-1}(y) \Rightarrow x \in N^{\prime-1}(y)$ for all $x, y \in U$ and thus, $N^{-1} \leq N^{\prime-1}$.

Hence, the comparability of the inverse operators follows immediately from the comparability of the thirteen original neighborhood operators. Based on Proposition 3 and Figure 1a, we obtain the lattice for the inverse neighborhood operators in Figure 1b. The goal is to combine Figures 1a and 1b. To this end, we need to study the comparability between the original neighborhood operators $A-M$ and the nine inverse neighborhood operators $A^{-1}-M^{-1}$. The comparability of operators $D, H, K$ and $L$ is already shown in Figure $1 \mathrm{~b}$, as it is a direct consequence of the symmetry of $N_{4}^{\mathbb{C}_{j}}$. Moreover, we have the following proposition:

Proposition 4. It holds that $A^{-1}, B^{-1}, C^{-1}, E^{-1}, F^{-1}, G^{-1}, M^{-1} \leq I$ and $A, B, C, E, F, G, M \leq I^{-1}$.

Proof. Immediate consequence from $H \leq I$ (Fig. 1a) and $H \leq I^{-1}$ (Fig. 1b).

Other partial order relations do not hold. In the next example we illustrate that the operator $A^{-1}$ is incomparable with $A$ and $B$. Other counterexamples can be established analogously.

Example 2. Let $U=\{1,2,3\}$ and $\mathbb{C}=\{\{3\},\{1,2\},\{1,3\},\{1,2,3\}\}$, then we compute that $N_{1}^{\mathbb{C}}(1)=N_{3}^{\mathbb{C}_{1}}(1)=\{1\}$ and $N_{1}^{\mathbb{C}}(2)=N_{3}^{\mathbb{C}_{1}}(2)=\{1,2\}$. However, $\left(N_{1}^{\mathbb{C}}\right)^{-1}(1)=\{1,2\}$ and $\left(N_{1}^{\mathbb{C}}\right)^{-1}(2)=\{2\}$. Hence, the neighborhood operators of $A^{-1}$ are incomparable with those of $A$ and $B$.

Therefore, we obtain the Hasse diagram of the twenty-two neighborhood operators of Table 2 in Figure 2. Note that there is a clear symmetry. The neighborhood operator $K$ yields the largest neighborhoods, while the operators $A$ and $A^{-1}$ yield the smallest.

\section{Hasse Diagram of Approximation Operators}

In this section, we discuss the Hasse diagram of different upper approximation operators defined in literature. In Section 4.1, we first discuss the element based approximation operators generated by the neighborhood operators stated in Table 2. In Section 4.2, we will discuss the partial order relation of other upper approximation operators which are not related with one of the neighborhood operators of Table 2 . 


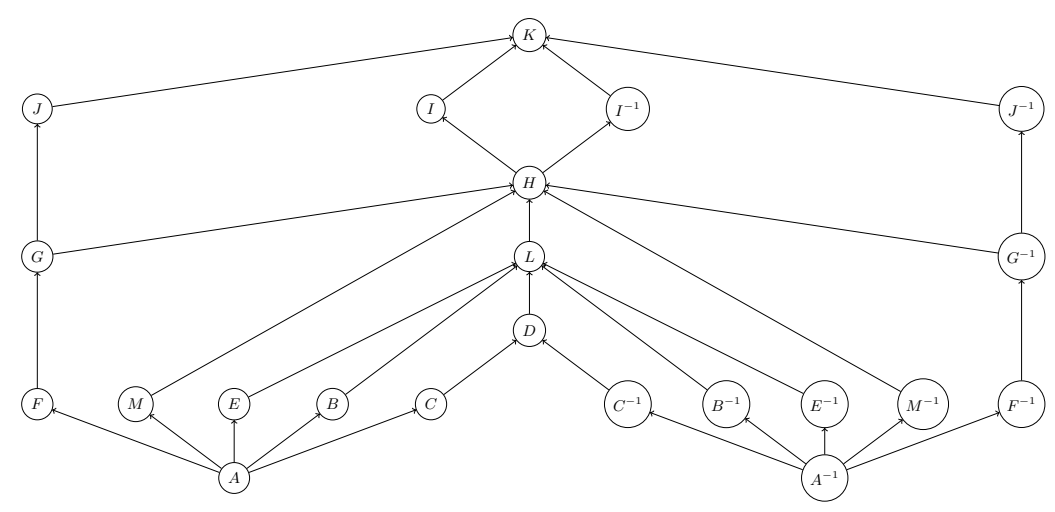

Fig. 2: Hasse diagram of thirteen neighborhood operators and their inverses

The study of the partial order relation of upper approximation operators gives an indication on the accuracy of the dual pair of approximation operators. This accuracy is often given by the ratio of the cardinality of the lower and upper approximation operator $\frac{|\mathrm{apr}|}{\mid \overline{|\overline{a p r}|}}([4])$. By duality it holds that

$$
\forall A \subseteq U: \underline{\operatorname{apr}}_{1}(A) \subseteq \underline{\operatorname{apr}}_{2}(A) \Leftrightarrow \overline{\operatorname{apr}}_{2}(A) \subseteq \overline{\operatorname{apr}}_{1}(A) .
$$

Therefore, smaller upper approximation operators yield higher accuracy.

\subsection{Element based Approximation Operators}

The results stated in Section 3.1 can be used to define new element based approximations operators, since they are only equal if the generating neighborhood operators are equal: given two neighborhood operators $N$ and $N^{\prime}$, then $\overline{\operatorname{apr}}_{N}=\overline{\operatorname{apr}}_{N^{\prime}}$ if and only if $N=N^{\prime}([3])$.

As discussed in Proposition 1, the approximation operators based on the neighborhood operators of groups $A^{-1}$ and $E^{-1}$ are $H_{5}^{\mathbb{C}}$ and $H_{3}^{\mathbb{C}}$, respectively. However, the approximation operators related to the other seven inverse neighborhood operators are not yet established in literature.

Moreover, the partial order relation between two neighborhood operators yields immediate results for the order relation between the corresponding approximation operators: if $N \leq N^{\prime}$, then for all $A \subseteq U, \overline{\operatorname{apr}}_{N}(A) \subseteq \overline{\operatorname{apr}}_{N^{\prime}}(A)$ ([7]). Therefore, the Hasse diagram of the upper approximation operators $\overline{\operatorname{apr}}_{N}$ is identical to the Hasse diagram of the neighborhood operators $N$ given in Figure 2.

From Figure 2, we derive that the approximation operators based on the neighborhood operators from groups $A$ and $A^{-1}$ have the highest accuracy amongst the element based approximation operators based on neighborhood operators from Table 2. Moreover, the approximation operators based on neighborhood operators from groups $B, C, E, F, M$ and their inverse operators have 


\begin{tabular}{|c|c|c|}
\hline Number & Lower & Upper \\
\hline 1 & $\operatorname{apr}_{\mathbb{C}}^{\prime}=\operatorname{apr}_{\mathbb{C}}^{\prime}$ & ${\overline{\mathrm{apr}_{\mathbb{C}}^{\prime}}}^{\prime}={\overline{\operatorname{apr}_{\mathbb{C}_{1}}}}^{\prime}$ \\
\hline 2 & $\mathrm{apr}_{\mathbb{C}_{2}}^{\prime}$ & $\overline{\mathrm{apr}}_{\mathbb{C}_{2}}^{\prime}$ \\
\hline 3 & ${\overline{\operatorname{apr}^{\prime}}}_{\mathbb{C}_{4}}^{\prime 2}$ & $\overline{\operatorname{apr}}_{\mathbb{C}_{4}}^{\prime}$ \\
\hline 4 & $\overline{\operatorname{apr}}_{\mathbb{C}_{n}}^{\prime 4}$ & $\overline{\operatorname{apr}}_{\mathbb{C}_{n}}^{\prime \prime}$ \\
\hline 5 & $\underline{\operatorname{apr}}_{S_{n}}$ & $\overline{\operatorname{apr}}_{S \cap}$ \\
\hline 6 & $\left(H_{1}^{\mathbb{C}}\right)^{\partial}$ & $H_{1}^{\mathbb{C}}$ \\
\hline 7 & $\left(H_{4}^{\mathbb{C}}\right)^{\partial}$ & $H_{4}^{\mathbb{C}}$ \\
\hline
\end{tabular}

Table 3: Dual covering based approximation operators

a high accuracy as well. Approximation operators based on $I, I^{-1}, J, J^{-1}$ and $K$ have a lower accuracy.

\subsection{Partial Order Relations of Other Approximation Operators}

In [3], the partial order relations with respect to other approximation operators than the element based approximation operators are discussed as well. These approximation operators are stated in Table 3. We shortly recall their definition here and study their partial relations with the approximation operators based on $B^{-1}, C^{-1}, F^{-1}, G^{-1}, I^{-1}, J^{-1}$ and $M^{-1}$. The partial order relations with the other element based approximation operators were already discussed in [3].

The approximation operators 1-4 are called tight covering based rough set approximation operators ([2]). The operator $\operatorname{apr}_{\mathbb{C}}^{\prime}$ is obtained by replacing the partition $U / E$ in Equation (1) by a covering $\overline{\mathbb{C}([5]): ~ l e t ~} A \subseteq U$, then

$$
\begin{aligned}
& \underline{\operatorname{apr}}_{\mathbb{C}}^{\prime}(A)=\bigcup\{K \in \mathbb{C} \mid K \subseteq A\}, \\
& \overline{\operatorname{apr}}_{\mathbb{C}}^{\prime}(A)=\operatorname{co}\left(\underline{\operatorname{apr}_{\mathbb{C}}^{\prime}}(\operatorname{co}(A))\right)=\{x \in U \mid(\forall K \in \mathbb{C})(x \in K \Rightarrow K \cap A \neq \emptyset)\} .
\end{aligned}
$$

Note that the tight covering based approximation operator based on $\mathbb{C}_{3}$ is equal to an element based approximation operator: $\underline{\operatorname{apr}}_{\mathbb{C}_{3}}^{\prime}=\underline{\operatorname{apr}}_{N_{1}^{\mathbb{C}}}([6])$.

The pair of approximation operators $\left(\operatorname{apr}_{S_{\cap}}, \overline{\operatorname{apr}}_{S_{\cap}}\right)$ is an example of a closure system based pair [10]. Let $S_{\cap, \mathbb{C}}$ be the intersection closure of the covering $\mathbb{C}$, i.e., the minimal subset of $\mathcal{P}(U)$ containing $\mathbb{C}, \emptyset$ and $U$ which is closed under set intersection, and let $S_{\cap, \mathbb{C}}^{\prime}=\left\{\operatorname{co}(K) \mid K \in S_{\cap, \mathbb{C}}\right\}$, then for $A \subseteq U$ it holds that

$$
\operatorname{apr}_{S_{\cap}}(A)=\bigcup\left\{K \in S_{\cap, \mathbb{C}}^{\prime} \mid K \subseteq A\right\}, \overline{\operatorname{apr}}_{S_{\cap}}(A)=\bigcap\left\{K \in S_{\cap, \mathbb{C}} \mid K \supseteq A\right\} .
$$

Finally, the operators $H_{1}^{\mathbb{C}}([12])$ and $H_{4}^{\mathbb{C}}([15])$ are defined by, for $A \subseteq U$,

$$
\begin{aligned}
& H_{1}^{\mathbb{C}}(A)=\underline{\operatorname{apr}}_{\mathbb{C}}^{\prime}(A) \cup\left(\bigcup\left\{\bigcup \operatorname{md}(\mathbb{C}, x): x \in A \backslash \underline{\operatorname{apr}}_{\mathbb{C}}^{\prime}(A)\right\}\right), \\
& H_{4}^{\mathbb{C}}(A)=\underline{\operatorname{apr}}_{\mathbb{C}}^{\prime}(A) \cup\left(\bigcup\left\{K \in \mathbb{C}: K \cap\left(A \backslash \underline{\operatorname{apr}_{\mathbb{C}}^{\prime}}(A)\right) \neq \emptyset\right\}\right),
\end{aligned}
$$




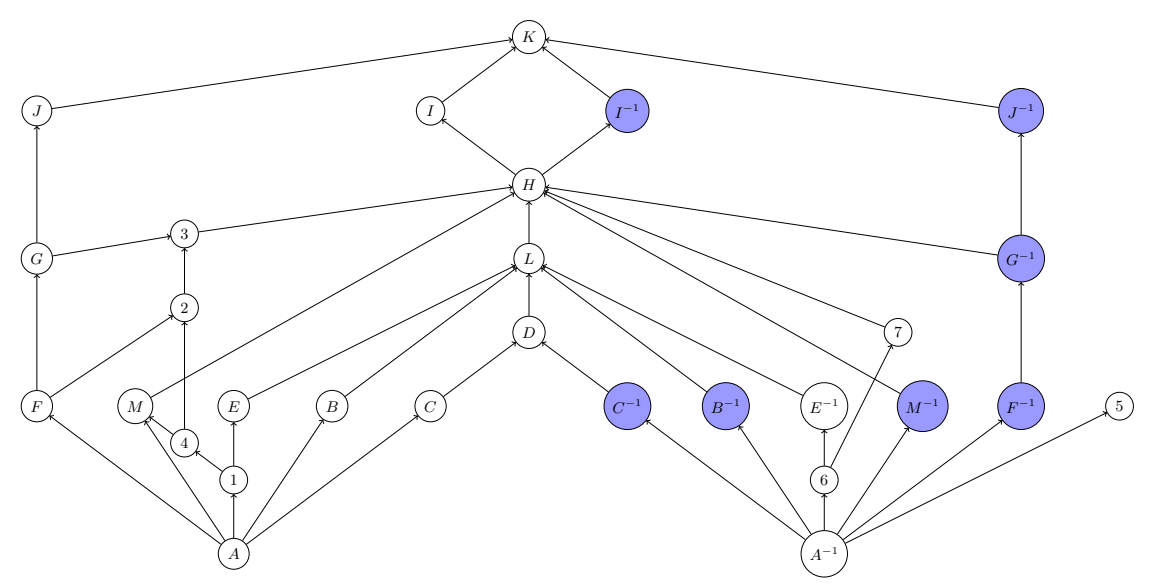

Fig. 3: Hasse diagram of twenty-nine upper approximation operators

and their dual lower approximation operators by $\left(H_{i}^{\mathbb{C}}\right)^{\partial}(A)=\operatorname{co}\left(H_{i}^{\mathbb{C}}(\operatorname{co}(A))\right)$. Both operators were proposed in the so-called non-dual framework of approximation operators [9].

In order to extend the Hasse diagram of the element based approximation operators with these seven other covering based approximation operators, we need to study the partial order relations between these seven operators, and the seven element based approximation operators based on the inverse neighborhood operators $B^{-1}, C^{-1}, F^{-1}, G^{-1}, I^{-1}, J^{-1}$ and $M^{-1}$. However, there are no new partial order relations than the ones established in Figure 2 and [3] as illustrated in the next example.

Example 3. Let $\mathbb{C}=\{\{1\},\{3\},\{1,3\},\{1,4\},\{2,4\},\{3,4\},\{2,3,4\}\}$ be a covering for $U=\{1,2,3,4\}$. Then $\overline{\operatorname{apr}}_{\left(N_{3}^{\mathbb{C}_{1}}\right)^{-1}}(\{1\})=\{1,4\}$ and $\overline{\operatorname{apr}}_{\left(N_{3}^{\mathbb{C}_{1}}\right)^{-1}}(\{4\})=$ $\{4\}$, but $\overline{\operatorname{apr}}_{\mathbb{C}}^{\prime}(\{1\})=\{1\}$ and $\overline{\operatorname{apr}}_{\mathbb{C}}^{\prime}(\{4\})=\{2,4\}$. Hence, $\overline{\operatorname{apr}}_{\left(N_{3}^{\left.\mathbb{C}_{1}\right)^{-1}}\right.}$ and $\overline{\operatorname{apr}}_{\mathbb{C}}^{\prime}$ are incomparable.

Therefore, we obtain the Hasse diagram of twenty-nine upper approximation operators in Figure 3, where we have highlighted the new rough set approximation operators. Observe that we denote the upper approximation operator $\overline{\operatorname{apr}}_{N}$ with the letter of the associated neighborhood operator $N$.

Concerning the accuracy of approximation operators, we see that the pairs of approximation operators which are not based on neighborhood operators (Table $3)$ achieve high accuracy in general, but lower accuracy than the approximation operators based on $A$ and $A^{-1}$.

\section{Conclusion and Future Work}

Neighborhood operators are fundamental in the research of covering based rough sets since they generalize the equivalence classes in Pawlak's original rough set 
model. In this article, we have established the Hasse diagram of twenty-two neighborhood operators, of which thirteen are obtained from the combinations

$N_{i}^{\mathbb{C}_{j}}$ and nine are inverse operators. Seven of these inverse neighborhood operators lead to new approximation operators.

Moreover, we have discussed the Hasse diagram of twenty-nine different upper approximation operators. Seven approximation operators are not related with neighborhood operators. The Hasse diagram of upper approximation operators enlightens the application perspective of approximation operators, since smaller upper approximation operators yield a higher accuracy. Therefore we conclude that not only element based approximation operators are useful for applications such as feature selection.

However, little research has been done on the application of non-neighborhoodbased rough set models in machine learning. Furthermore, the recent proliferation of approximation operators can bury the intuition to usefulness of the operators.

\section{Acknowledgements}

Lynn D'eer has been supported by the Ghent University Special Research Fund. Chris Cornelis was partially supported by the Spanish Ministry of Science and Technology under the project TIN2011-28488 and the Andalusian Research Plans P11-TIC-7765 and P10-TIC-6858, and by project PYR-2014-8 of the Genil Program of CEI BioTic GRANADA.

\section{References}

1. Z. Bonikowski, E. Brynarski, Extensions and intensions in rough set theory, Information Science 107 (1998) 149-167

2. I. Couso, D. Dubois, Rough sets, coverings and incomplete information, Fundamenta Informaticae 108 (2011) 223-247

3. L. D'eer, M. Restrepo, C. Cornelis, J. Gómez, Neighborhood operators for covering based rough sets, submitted

4. Z. Pawlak, Rough sets, International Journal of Computer and Information Sciences 11 (1982) 341-356

5. J.A. Pomykala, Approximation operations in approximation space, Bulletin de la Académie Polonaise des Sciences 35 (9-10), (1987) 653-662.

6. M. Restrepo, C. Cornelis, J. Gómez, Duality, conjugacy and adjointness of approximation operators in covering-based rough sets, International Journal of Approximate Reasoning 55 (2014) 469-485.

7. M. Restrepo, C. Cornelis, J. Gómez, Partial order relation for approximation operators in covering based rough sets, Information Sciences 284 (2014) 44-59.

8. E. Tsang, D. Chen, J. Lee, D.S. Yeung, On the upper approximations of covering generalized rough sets, Proceedings of the 3rd International Conference on Machine Learning and Cybernetics, (2004) 4200-4203.

9. T. Yang, Q. Li, Reduction about approximation spaces of covering generalized rough sets. International Journal of Approximate Reasoning, 51 (2010) 335-345. 
10. Y.Y. Yao, B. Yao, Covering based rough sets approximations. Information Sciences 200 (2012) 91-107.

11. Y. Y. Yao, Relational interpretations of neighborhood operators and rough set approximation operators, Information Sciences 101 (1998) 21-47

12. W. Żakowski, Approximations in the space $(u, \pi)$, Demonstratio Mathematica 16(1983) 761-769

13. W. Zhu, Topological approaches to covering rough sets, Information Sciences 177 (6) (2007) 1499-1508

14. W. Zhu, F. Wang, On three types of covering based rough sets. IEEE Transactions on Knowledge and Data Engineering, No. 8 (2007).

15. W. Zhu, F. Wang, A new type of covering rough sets. 3rd International IEEE Conference Intelligence Systems. (2006). 